\title{
Correction to: Superconvergence of Immersed Finite Volume Methods for One-Dimensional Interface Problems
}

\author{
Waixiang Cao ${ }^{1} \cdot$ Xu Zhang $^{2}$ (D) Zhimin Zhang Zh, $^{3,4}$ \\ Qingsong Zou ${ }^{5}$
}

Published online: 5 December 2017

(C) Springer Science+Business Media, LLC, part of Springer Nature 2017

\section{Correction to: J Sci Comput (2017) 73:543-565 https://doi.org/10.1007/s10915-017-0532-6}

The original version of this article unfortunately contained an error.

The affiliation of Dr. Qingsong Zou is incorrect. The correct affiliation is School of Data and Computer Science, Sun Yat-Sen University, Guangzhou, 51006, Guangdong, China.

The original article can be found online at https://doi.org/10.1007/s10915-017-0532-6.

Xu Zhang

xuzhang@math.msstate.edu

Waixiang Cao

ziye101@163.com

Zhimin Zhang

zmzhang@csrc.ac.cn

Qingsong Zou

mcszqs@mail.sysu.edu.cn

1 School of Mathematical Sciences, Beijing Normal University, Beijing 100875, China

2 Department of Mathematics and Statistics, Mississippi State University, Mississippi State, MS 39762, USA

3 Beijing Computational Science Research Center, Beijing 100193, China

4 Department of Mathematics, Wayne State University, Detroit, MI 48202, USA

5 School of Data and Computer Science, Sun Yat-Sen University, Guangzhou 51006, Guangdong, China 